\title{
Designing Pedibus Lines: a Path Based Approach
}

\author{
Federico Malucelli, Emanuele Tresoldi ${ }^{1}$ \\ Dipartimento di Elettronica, Informazione e Bioingegneria \\ Politecnico di Milano \\ Milano, Italy \\ Maddalena Nonato \\ Dipartimento di Ingegneria \\ Università degli Studi di Ferrara \\ Ferrara, Italy
}

\begin{abstract}
We study the problem of designing walking school bus lines (Pedibus) limiting the deviation with respect to the shortest path for each child, with the objective of minimizing the number of accompanying persons and the perceived risk of the selected trajectories. The problem is formulated using a path model and a column generation approach is proposed. Computational experiments compare the lower bounds and the solutions of the proposed approach with the arc model and a simple heuristic proposed in a previous work.
\end{abstract}

Keywords: Walking School Bus, Minimum leaf spanning tree with limited deviations, Column Generation.

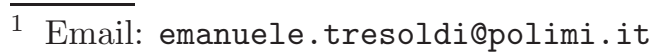




\section{Introduction}

Pedibus lines are an effective tool to educate young generations to an environmental sustainable behavior and to decrease the traffic congestion and air pollution in urban areas. Helping in designing efficient Pedibus lines is of great importance to propose an attractive system able to reach the objective. The problem can be described as follows: given the school location, the children home addresses, and the distance between each pair of locations, find the minimum number of lines rooted at the school so that each location belongs to one line and the distance from school to each location along the line is below a given threshold that depends on the length of actual shortest path. The objective function has two components: on the one hand it minimizes the number of supervising adults (one per line), on the other hand minimizes the perceived risk by favoring the merging of lines. We refer to this problem as Minimum Leaf Pedibus Line design Problem $(M L P L P)$. Looking at the problem in an abstract way, it recalls a minimum leaf spanning tree with a limit on the distance of each node from the root.

The Pedibus line design problem is usually approached in practice with common sense solutions, which are suitable in small contexts but are not able to manage the system on a large scale. It has some similarities with the well studied School Bus Routing problem, though peculiarities such as the tight constraint on deviations and the merging of lines make it different and justify a specific study to take advantage of them. The problem in this form is proposed and studied in [5] where a brief survey on the related literature is presented. That work proposes a simple arc model that fails to solve instances with more than 50 pupils and a simple heuristic that behaves reasonably well on large instances. In this work we build upon that experience and we directly compare with those results.

We propose an alternative path based formulation. For that formulation we describe a Column Generation approach to solve optimally the linear relaxation of the problem and generate a heuristic solution by solving the corresponding integer version of the master problem. The lower bounds and the feasible solutions obtained with this approach are compared with the solutions obtained with the simple arc model and the heuristic algorithm proposed in $[5]$. 


\section{Problem definition and path-based model for $M L P L P$}

The problem can be described considering a directed graph $G=(\mathcal{N}, \mathcal{A})$. The nodes in $\mathcal{N}$ represent walking-bus stops, that is children homes and the school (denoted by index 0 and called root), and the $\operatorname{arcs}$ in $\mathcal{A}$ correspond to shortest path connections between nodes. A coefficient $c_{i j}$ associated with every arc $(i, j) \in \mathcal{A}$ gives the length of the shortest path from $i$ to $j$, thus $c_{i 0}$ gives the length of the "ideal" path from node $i$ to the root. Another coefficient $d_{i j}$ gives the measure of the perceived risk traveling from $i$ to $j$, or alternatively the cost to adapt the trajectory from $i$ to $j$ to a given standard to avoid risks. Given a coefficient $\delta>1$, the problem consists in finding an arborescence routed in 0 and spanning all nodes in $\mathcal{N} \backslash\{0\}$ such that considering the path going from any node $i$ to 0 in the arborescence, its length is less than or equal to $\delta$ times the length of the ideal path. The objective is to minimize the number of leaves and also the sum of coefficients $d_{i j}$ for all arcs in the arborescence. Note that, in this representation, the leaves correspond to the accompanying persons and the paths converging to the root correspond to Pedibus lines. Lines can merge in some intermediate nodes to minimize the second term of the objective function.

The path model that we propose is based on the set $\mathcal{P}$ of all feasible paths. A path $p$ from any node $i$ to the root 0 is feasible if it is elementary (no two nodes are visited more than once) and its total length $c_{p} \leq \delta c_{i 0}$. To complete the notation let $\mathcal{P}_{i} \subset \mathcal{P}$ represent the subset of all paths visiting node $i \in \mathcal{N}$, $\mathcal{P}_{i j} \subset P$ the subset of all paths using arc $(i, j) \in A$ and $\overline{\mathcal{P}}_{i} \subset P$ the subset of all paths starting from node $i \in \mathcal{N}$.

The model makes use of two sets of binary variables: $x_{p}$ equal to 1 if path $p \in \mathcal{P}$ is selected and $y_{i j}$ equal to 1 if the $\operatorname{arc} \forall(i, j) \in \mathcal{A}$ is used. The complete formulation for the path-based MILP is given in (1-7).

The objective function (1) is made of two parts: minimization of the number of the selected paths, this corresponds to the minimization of the number of leaves, and minimization of total risk/cost multiplied by a suitable trade-off parameter $\epsilon$.

Constraint (2) imposes that all nodes have to be visited at least once. Inequality (3) defines the activation constraint for $y_{i j}$ variables. Constraint (4) limits the number of paths originating from a leaf to one. Finally, the last constraint (5) imposes that for each node but 0 a single outgoing arc must be selected, meaning that if two paths reach the same node they are merged together. 


$$
\begin{array}{lr}
\min & \sum_{p \in \mathcal{P}} x_{p}+\epsilon \sum_{(i, j) \in \mathcal{A}} d_{i j} y_{i j} \\
-\sum_{p \in P_{i}} x_{p} \leq-1 & \forall i \in \mathcal{N} \\
\sum_{p \in P_{i j}} x_{p}-|\mathcal{N}| y_{i j} \leq 0 & \forall(i, j) \in \mathcal{A} \\
\sum_{i \in \overline{\mathcal{P}}_{i}} x_{p} \leq 1 & \forall i \in \mathcal{N} \backslash\{0\} \\
\sum_{(i, j) \in \mathcal{A}} y_{i j}=1 & \forall i \in \mathcal{N} \backslash\{0\} \\
x_{p} \in\{0,1\} & \forall p \in \mathcal{P} \\
y_{i j} \in\{0,1\} & \forall(i, j) \in \mathcal{A} .
\end{array}
$$

Since the number of paths in $\mathcal{P}$ is exponential in $|\mathcal{N}|$ and we cannot explicitly generate all variables $x_{p}$ even for relatively small graphs, our solution approach is based on the column generation paradigm (see [1]).

The restricted master problem (RMP), given by the linear relaxation of (1-7), is initialized with either a set of $|\mathcal{N}|-1$ dummy columns representing the shortest paths from any node in $\mathcal{N} \backslash\{0\}$ to 0 or a set of columns corresponding to the heuristic solution provided by the greedy randomized algorithm described in [5].

In the pricing sub-problem we look for the most violated dual constraint (8) corresponding to the primal variable $x_{p}$ with the most negative reduced cost.

Let denote $\alpha, \beta$ and $\gamma$ as non-positive vectors of dual variables associated with constraints (2), (3) and (4) then the expression of the dual constraint associated with $x_{p}$ is:

$$
-\sum_{i \in \mathcal{N}_{p}} \alpha_{i}+\sum_{(i, j) \in \mathcal{A}_{p}} \beta_{i j}+\gamma_{h} \leq 1 \quad \forall p \in \overline{\mathcal{P}}_{h}, \forall h \in N \backslash\{0\}
$$

In practice, the pricing procedure has to find the feasible minimum cost path starting from any of the nodes in $\mathcal{N} \backslash\{0\}$ and arriving at the node 0 where the cost depends on $\alpha_{i}$ for each node $i \in \mathcal{N}$ and $\beta_{i j}$ for each $\operatorname{arch}(i, j) \in \mathcal{A}$. The total cost of a path $p$ is then defined as $r_{p}=\sum_{i \in \mathcal{N}_{p}} \alpha_{i}-\sum_{(i, j) \in \mathcal{A}_{p}} \beta_{i j}-\gamma_{h}$ where $h$ is the starting node of the path. If $r_{p}<1$ then the reduced cost of 
the variable $x_{p}$ associated with path $p$ is negative and $x_{p}$ is added to $\mathcal{P}$ in the RPM.

The problem of finding the feasible path with the most negative reduced cost can be seen as a resource constrained elementary shortest path problem (see [6] for a recent survey) in which the resource, that is monotonically consumed along the path, is the maximal allowed deviation from the shortest path $(\Delta)$.

In each iteration of the column generation procedure $|\mathcal{N}|-1$ independent pricing sub-problems, one for each node in $\mathcal{N} \backslash\{0\}$, are solved. Two different procedures are employed to tackle this problem. The first one is heuristic, it is based on a very fast nearest neighbor algorithm and it is mainly used to rapidly populate the set $\mathcal{P}$ in the first iterations of the column generation procedure. The second procedure solves the problem exactly using a slightly modified version of the pulse algorithm presented in [4], the procedure is executed every time the heuristic algorithm is not able to find any path with negative reduced cost. In our version of the pulse procedure the resource $\Delta$ is initialized to $\delta c_{h 0}$ where $h$ is the starting node of the path associated with that pricing sub-problem and it is reduced every time the path is extended. In detail, when the path is extended from node $i$, with resource value $\Delta_{i}$, to node $j$ the new value for the resource $\Delta_{j}$ is equal to $\min \left(\Delta_{i}-c_{i j}, \delta c_{j 0}\right)$. Moreover, denoted with $\Delta_{\min }$ and $\Delta_{\max }$ the minimum and the maximum values $\delta c_{i 0}$ $\forall i \in N \backslash\{0\}$, then the bounding procedure of the pulse algorithm is executed starting with a resource value equal to $\Delta_{\min }$ and it is increased by one tenth of $\Delta_{\max }-\Delta_{\min }$ in each iteration until $\Delta_{\max }$ is reached.

At the end of the column generation procedure an integer solution for the problem is achieved solving the MIP model (1-7) with all variables that has been included in $P$. Therefore, since we do not perform a full branch and price scheme the optimality of the integer solution cannot be guaranteed.

\section{Computational experiments}

In order to test our approach the column generation procedure has been implemented in Python 2.7 using Pyomo 5.0 as optimization modeling language (see [3] and [2]) and IBM CPLEX 12.7.0 as LP an MIP solver. Note that in our implementation of the pulse algorithm the use of multi-threading differs from the original. In particular, since for each iteration of column generation $|\mathcal{N}|-1$ independent pricing sub-problems have to be solved we prefer to solve all problems in parallel using a single-thread pulse implementation instead of solving pricing problems sequentially with a multi-thread algorithm. 
To provide a fare and coherent comparison between the path based and the arc based model, the testing campaign has been carried out on the same set of instances and on the same machine that were used to evaluate the performances of the arc based approach for $M L P L P$ (see [5]).

The results obtained are shown in table 1 . For each instance, columns in the table report: the number of nodes $|\mathcal{N}|$ (school excluded) in $G$, the maximum allowed $\delta$ value, the value of the linear relaxation of the arc based model (LP), the value of the linear relaxation computed with the column generation procedure (CG LP, * used when the optimum is reached), the value of the solution found by the greedy algorithm (Heur), the value of the best integer solution found by the arch based model without initialization and initialized with the greedy algorithm (MIP and HMIP, ${ }^{*}$ used when the optimal solution is reached), the value of the best integer solution found by the path based procedure initialized with dummy columns (CG MIP) and with the solution found by the greedy algorithm (HCG MIP), the percentage residual gap for the MIP model (1 - 7) solved with all the variables found in the column generation procedure initialized with the results of the greedy algorithm (G\% $\mathrm{HCG}$ ) and the total computational time that is the sum of execution time of the greedy algorithm, the column generation procedure (time-limit 1 hour) and the solving time of the resulting MIP (time-limit 1 hour plus what remains from the column generation procedure).

The LP relaxation provided by the column generation procedure is greatly superior to the linear relaxation of the arc based model. Indeed, the average gap between the CG LP and the corresponding HMIP on instances solved to the optimality is about $12 \%$ while it is more than $400 \%$ when the linear relaxation of the arc based model (LP column) is taken into account. However, computing LP CG can be time expensive and in 1 hour we are only able to compute the optimal continuous value of the RMP for graphs with less than 200 nodes. When $\delta$ is small $(1.1,1.2)$ this computational time is equally split between the solution of pricing sub-problems and the linear relaxation of the RMP. On the other hand, if delta is large then longer paths have to be considered and so the pricing phase is more time expensive and can take up to $80 \%$ of column generation procedure.

The integer solution found by the column generation procedure (CG MIP) is on average $13.76 \%$ better than the solution found by the arc based model (MIP). This value is greatly reduced, it is about $2.14 \%$, when both procedures are initialized with the results of the heuristic procedure (HMIP vs HCG MIP).

The greedy multi-start algorithm is very useful in particular in instances with more than 150 nodes and, when combined with the column generation 
procedure, provides solution that are on average $12.78 \%$ better than the solution found by the column generation procedure alone (HCG MIP vs CG MIP).

Considering only instances in which we are able to compute the optimal linear relaxation for the master problem (almost all instances with less than 200 nodes), the gap between CG LP and HCG MIP is about $13.58 \%$ while the final MIP gap, after 2 hours of computation, for the arc based model was about twice as much on the same instances (see [5] for detailed results).

Finally, a note on the computational time required by the MIP problem solved at the end of the column generation procedure for instances with more than 150 nodes. On the one hand, when $\delta$ is big the exact pricing procedure is extremely time consuming and can be executed only a few times (2 or 3 ) generating a small MIP problem that can be solved in a few seconds. On the other hand, when $\delta$ is small the pricing sub-problem can be solved more than 100 times and thousands of columns are added into the RMP. This generate a very large MIP problem that usually cannot be solved within 1 hour.

\section{References}

[1] Guy Desaulniers, Jacques Desrosiers and Marius M. Solomon, Column Generation, Springer, Boston, MA, (2005).

[2] William E. Hart, Carl D. Laird, Jean-Paul Watson, David L. Woodruff, Gabriel A. Hackebeil, Bethany L. Nicholson and John D. Siirola, Pyomo-optimization modeling in python, Springer Science \& Business Media, 67 (2017).

[3] William E. Hart, Jean-Paul Watson and David L. Woodruff, Pyomo: modeling and solving mathematical programs in Python, Mathematical Programming Computation, 3 (2011), 219-260.

[4] Leonardo Lozano, Daniel Duque and Andrs L. Medaglia, An Exact Algorithm for the Elementary Shortest Path Problem with Resource Constraints, Transportation Science, 50 (2016), 348-357.

[5] Federico Malucelli, Maddalena Nonato and Emanuele Tresoldi, Optimization based planning of Pedibus lines: an arc based approach, Transportation Research Procedia, 27 (2017), 760 - 767.

[6] Luigi Di Puglia Pugliese and Francesca Guerriero, A Survey of Resource Constrained Shortest Path Problems: Exact Solution Approaches, Networks, 62 (2013), $183-200$. 
Table 1

Path Model Results on Random Instances

\begin{tabular}{|c|c|c|c|c|c|c|c|c|c|c|}
\hline$|N|$ & $\delta$ & LP & CG LP & Heur & MIP & HMIP & CG MIP & HCG MIP & G\% HCG & $\mathrm{T}$ HCG \\
\hline 10 & 11 & 1.267 & $5.053^{*}$ & 5.713 & $5.571^{*}$ & $5.571^{*}$ & 5.571 & 5.571 & 0.000 & 1.025 \\
\hline 10 & 12 & 1.265 & $4.063^{*}$ & 4.693 & $4.693^{*}$ & $4.693^{*}$ & 4.693 & 4.693 & 0.000 & 0.830 \\
\hline 10 & 15 & 1.262 & $3.049^{*}$ & 3.576 & $3.482^{*}$ & $3.482^{*}$ & 3.482 & 3.482 & 0.000 & 1.422 \\
\hline 10 & 18 & 1.259 & $2.307^{*}$ & 3.562 & $3.418^{*}$ & $3.418^{*}$ & 3.418 & 3.418 & 0.000 & 1.524 \\
\hline 10 & 20 & 1.257 & $2.050^{*}$ & 3.590 & $2.550^{*}$ & $2.550^{*}$ & 2.550 & 2.550 & 0.000 & 2.433 \\
\hline 20 & 11 & 1.024 & $7.005^{*}$ & 7.124 & $7.114^{*}$ & $7.114^{*}$ & 7.114 & 7.114 & 0.000 & 1.814 \\
\hline 20 & 12 & 1.024 & $6.006^{*}$ & 7.120 & $6.115^{*}$ & $6.115^{*}$ & 6.115 & 6.115 & 0.000 & 3.082 \\
\hline 20 & 15 & 1.023 & $3.605^{*}$ & 5.098 & $4.084^{*}$ & $4.084^{*}$ & 4.086 & 4.086 & 0.000 & 4.969 \\
\hline 20 & 18 & 1.023 & $2.823^{*}$ & 3.097 & $3.090^{*}$ & $3.090^{*}$ & 3.094 & 3.097 & 0.000 & 2.431 \\
\hline 20 & 20 & 1.023 & $2.604^{*}$ & 3.102 & $3.071^{*}$ & $3.071^{*}$ & 3.087 & 3.081 & 0.000 & 2.872 \\
\hline 30 & 11 & 1.030 & $9.505^{*}$ & 10.191 & $10.143^{*}$ & $10.143^{*}$ & 10.151 & 10.150 & 0.000 & 5.599 \\
\hline 30 & 12 & 1.030 & $7.505^{*}$ & 9.175 & $8.132^{*}$ & $8.132^{*}$ & 8.137 & 8.137 & 0.000 & 9.461 \\
\hline 30 & 15 & 1.029 & $4.360^{*}$ & 5.155 & $5.109^{*}$ & $5.109^{*}$ & 5.129 & 5.129 & 0.000 & 26.700 \\
\hline 30 & 18 & 1.029 & $3.005^{*}$ & 4.151 & 4.097 & $3.146^{*}$ & 3.146 & 3.146 & 0.000 & 101.306 \\
\hline 30 & 20 & 1.029 & $2.694^{*}$ & 3.137 & 3.111 & 3.111 & 3.140 & 3.137 & 0.000 & 7.474 \\
\hline 50 & 11 & 1.031 & $12.006^{*}$ & 13.314 & $12.281^{*}$ & $12.281^{*}$ & 12.281 & 12.281 & 0.000 & 25.529 \\
\hline 50 & 12 & 1.031 & $9.450^{*}$ & 11.288 & $10.255^{*}$ & $10.255^{*}$ & 10.279 & 10.279 & 0.000 & 34.825 \\
\hline 50 & 15 & 1.030 & $6.783^{*}$ & 8.282 & 8.193 & 8.194 & 7.294 & 7.294 & 0.000 & 23.301 \\
\hline 50 & 18 & 1.030 & $4.719^{*}$ & 7.247 & 7.146 & 7.145 & 6.241 & 6.222 & 0.000 & 1364.550 \\
\hline 50 & 20 & 1.029 & $3.943^{*}$ & 6.243 & 6.148 & 6.150 & 5.234 & 4.261 & 0.000 & 2621.581 \\
\hline 80 & 11 & 1.034 & $9.364^{*}$ & 12.419 & 10.342 & 11.287 & 10.392 & 10.394 & 0.000 & 125.507 \\
\hline 80 & 12 & 1.033 & $7.235^{*}$ & 9.413 & 9.263 & 9.264 & 8.408 & 8.408 & 0.000 & 2491.820 \\
\hline 80 & 15 & 1.032 & $4.848^{*}$ & 6.409 & 7.183 & 6.269 & 7.395 & 6.409 & 0.000 & 92.759 \\
\hline 80 & 18 & 1.032 & $4.164^{*}$ & 5.375 & 8.131 & 5.236 & 7.368 & 5.375 & 0.000 & 137.700 \\
\hline 80 & 20 & 1.032 & $5.023^{*}$ & 5.380 & 8.123 & 5.202 & 7.398 & 5.380 & 0.000 & 324.440 \\
\hline 100 & 11 & 1.036 & $19.854^{*}$ & 24.590 & 21.488 & 21.486 & 21.522 & 21.533 & 0.000 & 146.327 \\
\hline 100 & 12 & 1.036 & $14.179^{*}$ & 18.561 & 17.367 & 17.384 & 16.486 & 15.536 & 0.000 & 374.995 \\
\hline 100 & 15 & 1.036 & $8.436^{*}$ & 11.522 & 15.226 & 10.387 & 11.505 & 10.497 & 11.930 & 7200.000 \\
\hline 100 & 18 & 1.036 & $6.393^{*}$ & 7.559 & 14.217 & 7.446 & 9.509 & 7.559 & 0.000 & 215.956 \\
\hline 100 & 20 & 1.036 & $6.105^{*}$ & 7.517 & 13.245 & 7.417 & 8.500 & 7.517 & 0.000 & 385.414 \\
\hline 150 & 11 & 1.004 & $16.491^{*}$ & 21.082 & 21.051 & 20.056 & 19.071 & 19.073 & 10.423 & 7200.000 \\
\hline 150 & 12 & 1.004 & 11.729 & 15.080 & 17.048 & 15.056 & 13.075 & 13.075 & 20.347 & 7200.000 \\
\hline 150 & 15 & 1.004 & $9.676^{*}$ & 10.078 & 18.035 & 10.067 & 15.077 & 10.078 & 0.000 & 746.739 \\
\hline 150 & 18 & 1.004 & $8.003^{*}$ & 8.076 & 21.027 & 8.070 & 16.081 & 8.076 & 0.000 & 1546.788 \\
\hline 150 & 20 & 1.004 & $7.003^{*}$ & 7.077 & 28.029 & 7.076 & 15.079 & 7.077 & 0.000 & 1483.544 \\
\hline 200 & 11 & 1.004 & $29.805^{*}$ & 38.119 & 33.080 & 33.081 & 31.105 & 31.105 & 0.000 & 843.554 \\
\hline 200 & 12 & 1.004 & 21.405 & 29.115 & 28.064 & 27.067 & 25.094 & 25.094 & 11.667 & 7200.000 \\
\hline 200 & 15 & 1.004 & 16.164 & 19.106 & 28.049 & 19.070 & 19.097 & 19.106 & 16.672 & 7200.000 \\
\hline 200 & 18 & 1.004 & 13.207 & 14.105 & 37.038 & 14.100 & 22.097 & 14.104 & 0.000 & 3684.441 \\
\hline 200 & 20 & 1.004 & 12.798 & 13.101 & 37.040 & 13.095 & 19.102 & 13.101 & 0.000 & 3632.482 \\
\hline 250 & 11 & 1.005 & 31.346 & 43.140 & 39.088 & 38.093 & 36.127 & 36.127 & 0.000 & 7200.000 \\
\hline 250 & 12 & 1.005 & 24.531 & 32.131 & 35.068 & 31.080 & 31.130 & 32.127 & 20.272 & 7200.000 \\
\hline 250 & 15 & 1.005 & 18.742 & 20.130 & 31.065 & 20.114 & 30.125 & 20.129 & 0.000 & 3657.909 \\
\hline 250 & 18 & 1.005 & 15.162 & 16.124 & 48.062 & 16.119 & 26.131 & 16.122 & 0.000 & 3688.617 \\
\hline 250 & 20 & 1.005 & 13.004 & 13.125 & 48.061 & 13.120 & 25.131 & 13.125 & 0.000 & 3643.244 \\
\hline 300 & 11 & 1.005 & 33.737 & 44.165 & 43.096 & 41.096 & 37.147 & 37.147 & 8.035 & 7200.000 \\
\hline 300 & 12 & 1.005 & 25.848 & 35.156 & 41.075 & 34.107 & 43.149 & 35.156 & 7.500 & 7200.000 \\
\hline 300 & 15 & 1.005 & 21.004 & 21.150 & 53.075 & 21.148 & 38.148 & 21.150 & 0.000 & 3671.378 \\
\hline 300 & 18 & 1.005 & 14.004 & 14.150 & 61.064 & 14.149 & 36.143 & 14.150 & 0.000 & 3658.683 \\
\hline 300 & 20 & 1.005 & 13.004 & 13.148 & 82.068 & 13.145 & 35.156 & 13.148 & 0.000 & 3687.936 \\
\hline
\end{tabular}

